\title{
Review of Rifaximin: A Summary of the Current Evidence and Benefits Beyond Licensed Use
}

Authors:

Disclosure:

Acknowledgements:

Received:

Accepted:

Keywords:

Citation:
Rooshi Nathwani, Benjamin Mullish, David Kockerling, Alexander Cole, Nowlan Selvapatt, *Ameet Dhar

Liver Unit/Division of Digestive Diseases, Department of Metabolism, Digestion and Reproduction, Imperial College London, UK

*Correspondence to a.dhar@imperial.ac.uk

The authors have declared no conflicts of interest

The division receives financial support from the National Institute of Health Research (NIHR) Biomedical Research Centre based at Imperial College Healthcare NHS Trust and Imperial College London. Mullish is the recipient of an NIHR Academic Clinical Lectureship (CL-2019-21-002).

03.02.21

22.02 .21

Antibiotics, hepatic encephalopathy, infection, rifaximin.

EMJ. 2021;6[3]:94-99.

\section{Abstract}

Antibiotic resistance in patients with cirrhosis continues to draw significant attention. With a propensity to frequent hospitalisations, patients with cirrhosis are subject to frequent antibiotic prescription. This increases their risk of developing resistance to one or more antimicrobial agents, making management of their condition particularly challenging. Despite advancements being made in the management of liver disease, mortality rates continue to rise: almost 5 -fold in those $<65$ years of age while remaining the leading cause of death in those 35-49 years of age. Alternative therapeutic options to prevent disease progression and cirrhosis-associated complications are urgently required; rifaximin is one such example. The medication use in patients with cirrhosis demonstrates additional benefits beyond current licensed use in the UK, that being for the prevention of hepatic encephalopathy and traveller's diarrhoea; rifaximin has especially been explored beyond current licensed use in the context of entericdriven pathologies. Through the therapy's key central action as a broad-spectrum antimicrobial, rifaximin has the ability to modulate the gut-liver axis via removal of gut microbial products associated with the progression of cirrhosis and its sequalae.

The benefits of rifaximin use continues to gather momentum, given its non-absorbable nature and well-tolerated side-effect profile, and these require consideration. With broad-spectrum antimicrobial properties, its use may assist in overcoming the conundrum posed of antibiotic resistance amongst patients with cirrhosis. This literature review discusses the chemical and antimicrobial properties of rifaximin, its licenced indication for use, and its reported benefits beyond this, as well as concerns regarding rifaximin resistance. 


\section{INTRODUCTION}

Liver disease constitutes a major global health burden, with mortality rates increasing 4-fold since the 1970s and 5-fold in those <65 years of age. Liver disease represents the leading cause of death in those 35-49 years of age, and the third most common cause of death in those 50-64 years of age in 2019.' Its rising incidence and rate of prevalence are largely attributable to risk factors for the most common conditions, including growing levels of alcohol consumption, the obesity epidemic, and viral hepatitis. ${ }^{2,3}$

Cirrhosis remains the primary driver of liverrelated deaths. Pathologically, progression from inflammation to fibrosis and on to cirrhosis occurs as a result of continual exposure to a hepatic insult. ${ }^{4}$ Treating the underlying aetiology of liver disease can slow progression, although in some cases the only cure remains liver transplantation; however, this is restricted to selected patients only and is further limited by organ supply. ${ }^{5}$ This highlights the urgent need to explore novel therapeutic options that ameliorate disease progression and its complications.

As a result of progressive immune dysfunction, patients with cirrhosis are at risk of recurrent bacterial infections, which are recognised as a major cause for the development of liver-related complications, advancement of liver failure, and repeated hospitalisations. ${ }^{6}$ These patients are frequently prescribed antibiotics as treatment or for infection prophylaxis, making them susceptible to developing multiple drug-resistant and extensively drug-resistant organisms, for which limited therapeutic options exist.,8 This review discusses the growing understanding of the possible role of rifaximin as an agent that may impact upon the trajectory of chronic liver disease, as a tool in the prevention of antibiotic resistance in chronic liver disease, and other potential areas of clinical benefit.

\section{RIFAXIMIN}

The use of rifaximin beyond its current licensed indications has been explored, especially in the context of enteric-driven pathologies. Translation into clinical practice has been hindered by a paucity of robust clinical trials. A considered review of the current understanding and uncertainties surrounding rifaximin use in decompensated cirrhosis is needed to inform appropriate future trial design, and therefore is discussed further here.

\section{Chemical Structure}

Rifaximin is a semi-synthetic, non-aminoglycoside oral antibiotic derived from rifamycin. ${ }^{9}$ Its pyridoimidazole ring differentiates it from other rifamycin derivates and leaves rifaximin waterinsoluble, therefore inhibiting absorption in the gastrointestinal tract.10,11 Its molecular mass size of 789.9 Da enables self-association, further preventing systemic absorption. ${ }^{12}$ Consequently, adjustments of dosing are not required. ${ }^{13}$ Following oral administration, the concentration of detectable rifaximin in blood and urine is less than $0.4 \%$, but up to $97.0 \%$ in stool, suggesting that its bioavailability is localised to the gastrointestinal tract. ${ }^{14}$

\section{Antimicrobial Properties}

Rifaximin inhibits bacterial RNA synthesis through its action on the $\beta$-subunit of bacterial DNAdependent RNA polymerase. ${ }^{15}$ In vitro studies have demonstrated that rifaximin has antibiotic coverage against Gram-positive and Gramnegative aerobic and anaerobic bacteria (Table 1). It also shows effectiveness against the protozoa Cyrptosporidium parvuum and Blastocystis hominis. ${ }^{17} \quad$ Rifaximin has demonstrated intermediate in vitro activity against various enteropathogens, with minimum inhibitory concentrations $(\mathrm{MIC})_{50}$ and $\mathrm{MIC}_{90}$ values ranging 8-64 and $16-128 \mu \mathrm{g} / \mathrm{mL}$, respectively. ${ }^{18,19}$ Yet, rifaximin achieves potent in vivo activity against enteropathogens, which can be explained by its extremely high luminal concentrations (4,000$8,000 \mu \mathrm{g} / \mathrm{g}$ ) that far exceed $\mathrm{MIC}_{90}$ values. ${ }^{18,20}$ Certain enteric bacteria, such as Clostridium difficile, have shown increased susceptibility to rifaximin with lower $\left.\mathrm{MIC}_{50}(\leq 0.25 \mu \mathrm{g} / \mathrm{mL})\right)^{16,18}$

The solubility of rifaximin is $70-120$ times greater in bile than in aqueous solution. This aids action in the small intestine which is bile-rich, compared to the aqueous colonic environment. ${ }^{21}$ Encouragingly, rifaximin does not significantly alter the composition of the gut microbiota but rather selectively targets bacteria (Table 1).

Concurrently, rifaximin favours the growth of beneficial bacterial species. ${ }^{22}$ 
Table 1: Isolated bacteria for which rifaximin has anti-microbial activity. . $^{13,16}$

\begin{tabular}{|c|c|c|}
\hline & Gram positive & Gram negative \\
\hline Aerobic Bacteria & $\begin{array}{l}\text { Enterococcus } \\
\text { Mycobacterium tuberculosis } \\
\text { Streptococcus pyogenes } \\
\text { Enterococcus faecalis } \\
\text { Streptococcus pneumoniae } \\
\text { Staphylococcus epidermidis } \\
\text { Staphylococcus aureus }\end{array}$ & $\begin{array}{l}\text { Escherichia coli } \\
\text { Shigella spp } \\
\text { Salmonella spp } \\
\text { Yersinia enterocolitica } \\
\text { Proteus spp } \\
\text { Peptococcus } \\
\text { Peptostreptococcus spp } \\
\text { Vibrio cholerae }\end{array}$ \\
\hline Anaerobic Bacteria & $\begin{array}{l}\text { Clostridium perfringens } \\
\text { Clostridioides difficile } \\
\text { Peptococcus } \\
\text { Peptostreptococcus } \\
\text { Bifidobacterium spp }\end{array}$ & $\begin{array}{l}\text { Bacteroides bivius-diseiens } \\
\text { Bacteroides fragilis } \\
\text { Helicobacter pylori } \\
\text { Fusobacterium spp } \\
\text { Klebsiella pneumoniae }\end{array}$ \\
\hline
\end{tabular}

\section{Indications for Rifaximin Use}

In view of its enterically localised bioavailability, rifaximin has primarily complemented the management of gastrointestinal pathology: travellers' diarrhoea, infectious diarrhoea, small intestinal bacterial overgrowth (SIBO), irritable bowel syndrome, diverticular disease, infection prophylaxis post-colorectal surgery, and inflammatory bowel disease. ${ }^{23}$ Over the last decade it has also emerged as a key therapeutic agent in the management of hepatic encephalopathy (HE). ${ }^{24}$ Despite widespread use in Europe for the above conditions, ${ }^{25}$ rifaximin is licensed only to minimise recurrent episodes of overt $\mathrm{HE}$ (high-quality evidence, strong recommendation $)^{26}$ and to treat travellers' diarrhoea in the UK and USA (high-quality evidence, strong recommendation), ${ }^{27}$ as well as to manage irritable bowel syndrome with diarrhoea in the USA (moderate-quality evidence, conditional recommendation). ${ }^{28-30}$

\section{Hepatic encephalopathy}

Rifaximin improves the severity of acute and chronic HE by targeting deaminating enteric bacteria producing systemically absorbed nitrogenous compounds, including ammonia. ${ }^{31}$ Concurrently, rifaximin reduces pathogenic bacterial species, thereby reconditioning the gut microbiome and reducing systemic endotoxaemia. ${ }^{32}$

The beneficial effects of rifaximin in the treatment of overt HE were initially established in a doubleblind, placebo-controlled trial by Bass et al., ${ }^{24}$ demonstrating that rifaximin was associated with a 6-month risk reduction in episodes of overt $\mathrm{HE}$ and HE-associated hospitalisations. ${ }^{24}$ In a further randomised controlled trial ( $R C T$ ), rifaximin plus lactulose demonstrated superiority over lactulose alone in decreasing hospital stay and all-cause mortality in patients with decompensated cirrhosis (23.8\% versus $49.1 \%$; $p<0.05$ ). ${ }^{33}$ These results were reinforced by a meta-analysis of 19 
RCTs including 1,370 patients. ${ }^{34}$ Rifaximin use for HE in clinical practice is further advocated by its short- and long-term safety profiles. Its tolerance is well acknowledged, especially when compared to alternative treatment options. ${ }^{35,36}$ Furthermore, its demonstrated benefits are beyond the physical alone, and it significantly improves health-related quality of life of patients with cirrhosis with $\mathrm{HE} .{ }^{37}$ In light of this, rifaximin is recommended in national and international consensus guidelines as an effective adjunct to lactulose for the prevention of recurrent overt HE. ${ }^{38}$

\section{Other Potential Benefits of Rifaximin}

Rifaximin has been shown to effect bacterial translocation, thereby modulating the gut microbiome. Therefore, it has the potential to modify the interplay of the gut-liver axis, central to progression of cirrhosis and its sequelae. ${ }^{39}$ Data to support this have been reported, though changes to guidelines are yet to be implemented.

\section{Spontaneous bacterial peritonitis}

With guidelines recommending the use of norfloxacin for the management of spontaneous bacterial peritonitis (SBP), Campillo et al. ${ }^{40}$ described the effects of long-term antibiotic administration, namely increased severity of hospital-acquired, Gram-positive staphylococcal infections and quinolone resistance. Presently, antibiotic resistance poses a significant challenge in the management of infection in patients with cirrhosis, with a pressing need for alternative prophylactic antibiotics.

Organisms responsible for the development of SBP are both Gram-negative and Grampositive. Frequently isolated Gram-negative pathogens include Escherichia coli, Klebsiella pneumoniae, and Streptococcus pneumoniae. ${ }^{41}$ Gram-positive pathogens implicated include methicillin-resistant Staphylococcus aureus and enterococci. ${ }^{42}$ As shown in Table 1, rifaximin demonstrated antimicrobial effectiveness against all these pathobionts and so its use in preventing SBP has been previously investigated.

In an RCT comparing rifaximin to norfloxacin in 262 patients affected by cirrhosis, rifaximin was superior in reducing the incidence of recurrent SBP over 6 months (3.88\% versus 14.13\%; $\mathrm{p}=0.04) .^{43}$ Similar findings were established for the primary prevention of SBP in patients with hepatitis-C-induced cirrhosis. ${ }^{44}$ In a metaanalysis comparing rifaximin to various systemic antibiotics, rifaximin lowered SBP risk by $47 \%$ compared to placebo for primary prevention, and by $74 \%$ compared to systemic antibiotics for secondary prevention. ${ }^{45}$ Prospective open-label studies have been less optimistic, demonstrating mixed results that concluded rifaximin did not consistently or reliably prevent SBP. ${ }^{46}$ Very few studies have assessed secondary SBP occurrence in patients with cirrhosis, concomitantly prescribed antibiotic prophylaxis for SBP and rifaximin for HE. Consequently, rifaximin alone is not recommended for the treatment or prevention of SBP as per consensus guidelines. ${ }^{8}$

\section{Endotoxaemia and haemodynamics}

Endotoxins IL-6 and TNF-a have been shown to be associated with development of cirrhosis-associated immune dysfunction, hyperdynamic circulation, and multi-organ dysfunction. These endotoxins can potentially be influenced by rifaximin use. Kalambokis et al. ${ }^{47}$ demonstrated in 13 patients with advanceddisease cirrhosis that rifaximin use reduced IL-6 and TNF-a, subsequently improving systemic haemodynamics, cardiac output, and plasma renin activity. ${ }^{47}$ Furthermore, 28 days of rifaximin administration was shown to significantly lower plasma endotoxin levels and hepatovenous pressure gradient measurements, independent of $\beta$-blocker use. ${ }^{48}$ After 5 -year follow-up of the same cohort, the rifaximin-treated cohort demonstrated reduced complication rates of variceal haemorrhage, hepatorenal syndrome, and SBP, as well as survival benefit compared to the control group. ${ }^{49}$ Rifaximin modulates gut epithelial physiology and prevents bacterial adherence and internalisation, reducing the release of pro-inflammatory cytokines (IFN- $\mathrm{y}$, IL-4, IL-6, IL-8, IL-12, and VCAM-1) implicated in cirrhosis-associated immune dysfunction..$^{50}$ Partly responsible for this phenomenon is the rifaximin-induced activation of the gut-specific pregnane- $X$ receptor, ${ }^{18}$ thereby suppressing NF-KB signalling, a pathway responsible for pro-inflammatory cytokine release. ${ }^{51}$ This may help to reduce intestinal permeability, a recognised process contributing to infections in cirrhosis. ${ }^{18}$ 


\section{Small intestinal bacterial overgrowth}

The role of SIBO in bacterial infection in cirrhosis is well recognised. ${ }^{6}$ Treatment for this condition is largely based on antibiotic therapy, for which rifaximin is one efficacious option. ${ }^{52}$ In a meta-analysis of 32 studies and 1,331 patients, rifaximin treated SIBO in $70.8 \%$ of patients (95\% confidence interval: 61.4-78.2). ${ }^{53}$ SIBO occurs frequently in cirrhosis, but few studies have interrogated antibiotic effectiveness in this cohort. One small-scale study by Zhang et al. ${ }^{54}$ reported that rifaximin was clinically effective in treating SIBO in $76 \%$ of patients with a confirmed diagnosis of cirrhosis.

\section{Cirrhosis-associated morbidity}

Recent evidence has proposed that rifaximin use may reduce complications associated with cirrhosis. In a Phase III RCT, 6-month-long rifaximin administration reduced the relative risk of a first cirrhosis-associated complication by $59 \%$ in patients with advanced disease. ${ }^{55}$ In a retrospective analysis of 101 patients awaiting liver transplantation, rifaximin increased the time interval to readmission and reduced incidence of portal hypertensive complications: variceal bleeding, complications of ascites, and hepatorenal syndrome. Patients receiving rifaximin exhibited a survival benefit and were less likely to be prioritised for organ transplantation, highlighting the benefits of rifaximin. ${ }^{56,57}$

In addition to clinical benefits, rifaximin therapy may have wider socio-economic advantages, especially in limited resource settings. In a UKbased retrospective study, rifaximin significantly reduced 30-day readmissions and emergency department attendances, as well as hospital and critical care bed days, 6 and 12 months post-commencement of treatment, ${ }^{58}$ incurring annual savings of 1,480-3,228 GBP per patient. ${ }^{59}$

\section{RIFAXIMIN RESISTANCE}

Until recently, resistance to rifaximin was thought to be uncommon.60 This perception changed when a mutation was identified in the $r p o \beta$ gene.
This encodes the target site of rifaximin, giving rise to concerns regarding the emergence of resistance in patients with cirrhosis on longterm rifaximin treatment. ${ }^{13,61}$ These concerns were substantiated by an observational study of 388 patients with cirrhosis, of whom 46 (11.9\%) developed C. difficile infection. Importantly, $30.4 \%$ of those who developed C. difficile infection were established on rifaximin for $\mathrm{HE}$ prophylaxis. Overall, C. difficile resistance to rifaximin was observed in $34.1 \%$ of cases and in $84.6 \%$ of patients who had previously received rifaximin. These findings stand in contrast to previous clinical studies and in vitro data, in which rifaximin demonstrated good antibacterial activity against $C$. difficile and in which the emergence of resistant clones was rare. ${ }^{62}$ With resistance patterns being considerable in those with previous and no previous exposure, rifaximin resistance may be an emerging problem in cirrhosis and will require further investigation.

\section{CONCLUSION}

While it is currently licensed for the treatment of recurrent episodes of HE, the full therapeutic potential of rifaximin in liver disease may be underutilised. Rifaximin displays broad-spectrum antimicrobial activity against pathobiont enteric bacteria and demonstrates anti-inflammatory properties. It also has the ability to modulate the gut microbiome, thereby preventing bacterial translocation. ${ }^{18}$ These processes are fundamental in the pathogenesis of cirrhosisassociated infection and therefore rifaximin use may be effective in this scenario. ${ }^{6}$ However, there remains a lack of robust evidence in the form of adequately powered RCTs to translate these perceived benefits into clinical practice. To date, studies have focused only on the prevention of SBP, rather than all infections. There remains uncertainty regarding rifaximin resistance; yet, based on the current evidence, rifaximin use beyond the licensed indications may be a significant step forward in the management and prevention of cirrhosis-associated morbidity and mortality. 


\section{References}

1. Office for National Statistics. Deaths registered in England and Wales. 2019. Available at: https://www.ons. gov.uk/peoplepopulationandcommunity/birthsdeathsandmarriages/ deaths/datasets/deathsregisteredinenglandandwalesseriesdrreferencetables. Last accessed: 16 November 2016.

2. Williams R et al. Addressing liver disease in the UK: a blueprint for attaining excellence in health care and reducing premature mortality from lifestyle issues of excess consumption of alcohol, obesity, and viral hepatitis. Lancet. 2014;384(9958):1953-97.

3. Ratib S et al. Liver cirrhosis in England - an observational study: are we measuring its burden occurrence correctly? BMJ Open. 2017;7(7):e013752

4. Roth GA et al.; GBD 2017 Causes of Death Collaborators. Global, regional, and national age-sex-specific mortality for 282 causes of death in 195 countries and territories, 19802017: a systematic analysis for the Global Burden of Disease Study 2017 Lancet. 2018;392(10159):1736-88.

5. Blachier $M$ et al. The burden of liver disease in Europe: a review of available epidemiological data. J Hepatol. 2013;58(3):593-608.

6. Jalan $\mathrm{R}$ et al. Bacterial infections in cirrhosis: a position statement based on the EASL Special Conference 2013. J Hepatol. 2014;60(6):1310-24.

7. Fernández $\mathrm{J}$ et al. The challenges of multi-drug-resistance in hepatology. $J$ Hepatol. 2016;65(5):1043-54.

8. Angeli et al.; European Association for the Study of the Liver. EASL Clinical Practice Guidelines for the management of patients with decompensated cirrhosis. J Hepatol. 2018;69(2):406-60.

9. Scarpignato C, Pelosini I. Experimental and clinica pharmacology of rifaximin, a gastrointestinal selective antibiotic. Digestion. 2006;73(Suppl 1):13-27.

10. Marchi E et al. 4-Deoxypyrido[1',2':1,2] imidazo[5,4-c]rifamycin SV derivatives. A new series of semisynthetic rifamycins with high antibacterial activity and low gastroenteric absorption. J Med Chem. 1985;28(7):960-3.

11. Baker DE. Rifaximin: a nonabsorbed oral antibiotic. Rev Gastroenterol Disord. 2005;5(1):19-30.

12. jetti $V$ et al. Rifaximin pharmacology and clinical implications. Expert Opin Drug Metab Toxicol. 2009;5(6):67582.

13. Koo HL, DuPont HL. Rifaximin: a unique gastrointestinal-selective antibiotic for enteric diseases. Curr
Opin Gastroenterol. 2010;26(1):17-25.

14. Descombe JJ et al. Pharmacokinetic study of rifaximin after oral administration in healthy volunteers. Int J Clin Pharmacol Res. 1994;14(2):51-6.

15. Gillis JC, Brogden RN. Rifaximin. A review of its antibacterial activity, pharmacokinetic properties and therapeutic potential in conditions mediated by gastrointestinal bacteria. Drugs. 1995;49(3):467-84.

16. Pimentel et al. Repeat rifaximin for irritable bowel syndrome: no clinically significant changes in stool microbial antibiotic sensitivity. Dig Dis Sci. 2017;62(9):2455-63.

17. Rivkin A, Gim S. Rifaximin: new therapeutic indication and future directions. Clin Ther. 2011;33(7):812-27.

18. Calanni F et al. Rifaximin: beyond the traditional antibiotic activity. J Antibiot (Tokyo). 2014;67(9):667-70

19. Sierra JM et al. In vitro activity of rifaximin against enteropathogens producing traveler's diarrhea. Antimicrob Agents Chemother. 2001;45(2):643-4.

20. DuPont $\mathrm{HL}$ et al. Rifaximin versus ciprofloxacin for the treatment of traveler's diarrhea: a randomized, double-blind clinical trial. Clin Infect Dis. 2001;33(11):1807-15.

21. DuPont HL. Biologic properties and clinical uses of rifaximin. Expert Opin Pharmacother. 2011;12(2):293-302.

22. Ponziani FR et al. Eubiotic properties of rifaximin: disruption of the traditional concepts in gut microbiota modulation. World J Gastroenterol. 2017;23(25):4491-9.

23. Su CG et al. Utility of the nonabsorbed (<0.4\%) antibiotic fifaximin in gastroenterology and hepatology. Gastroenterol Hepatol (NY). 2006;2(3): 186-97.

24. Bass NM et al. Rifaximin treatment in hepatic encephalopathy. N Engl J Med. 2010;362(12):1071-81.

25. Jiang ZD, DuPont HL. Rifaximin: in vitro and in vivo antibacterial activity - a review. Chemotherapy. 2005;51(Suppl 1):67-72

26. Vilstrup $\mathrm{H}$ et al. Hepatic encephalopathy in chronic liver disease: 2014 Practice Guideline by the American Association for the Study Of Liver Diseases and the European Association for the Study of the Liver. Hepatology. 2014;60(2):715-35.

27. Riddle MS et al. ACG Clinical Guideline: diagnosis, treatment, and prevention of acute diarrheal infections in adults. Am J Gastroenterol. 2016;111(5):602-22.

28. Weinberg DS et al.; American
Gastroenterological Association. American Gastroenterological Association Institute Guideline on the pharmacological management of irritable bowel syndrome. Gastroenterology. 2014;147(5): 1146-8.

29. U.S. Food and Drug Administartion (FDA). Full Prescribing Information. 1 Indications and Usage. 2004. Available at: https://www.accessdata. fda.gov/drugsatfda_docs/ label/2017/021361s023lbl.pdf. Last accessed: 24 February 2021.

30. National Institute for Health and Care Excellence (NICE). Rifaximin for preventing episodes of enting episodes of overt hepatic encephalopathy (TA337). 2015. Available at: https://www.nice.org. uk/guidance/ta337/resources/ rifaximin-for-preventing-episodesof-overt-hepatic-encephalopathypdf-82602552414661. Last accessed: 24 February 2021.

31. Flamm SL. Rifaximin treatment for reduction of risk of overt hepatic encephalopathy recurrence. Therap Adv Gastroenterol. 2011;4(3):199-206.

32. Tapper EB et al. Refining the ammonia hypothesis: a physiologydriven approach to the treatment of hepatic encephalopathy. Mayo Clin Proc. 2015;90(5):646-58.

33. Sharma BC et al. A randomized, double-blind, controlled trial comparing rifaximin plus lactulose with lactulose alone in treatment of overt hepatic encephalopathy. Am J Gastroenterol. 2013;108(9):1458-63.

34. Kimer $\mathrm{N}$ et al. Systematic review with meta-analysis: the effects of rifaximin in hepatic encephalopathy. Aliment Pharmacol Ther. 2014;40(2):123-32.

35. Maclayton DO, Eaton-Maxwell A Rifaximin for treatment of hepatic encephalopathy. Ann Pharmacother 2009;43(1):77-84.

36. Mullen KD et al. Rifaximin is safe and well tolerated for long-term maintenance of remission from overt hepatic encephalopathy. Clin Gastroenterol Hepatol. 2014;12(8):1390-7.e2.

37. Sanyal A et al. Randomised clinical trial: rifaximin improves health-related quality of life in cirrhotic patients with hepatic encephalopathy - a double-blind placebo-controlled study. Aliment Pharmacol Ther. 2011;34(8):853-61.

38. Vilstrup $\mathrm{H}$ et al.; American Association for the Study of Liver Diseases; European Association for the Study of Liver. Hepatic encephalopathy in chronic liver disease: 2014 practice guideline by the European Association for the Study of the Liver and the American Association for the Study of Liver Siseases. J Hepatol. 2014;61(3):64259. 
39. Fukui H. Gut-liver axis in live cirrhosis: how to manage leaky gut and endotoxemia. World J Hepatol. 2015;7(3):425-42.

40. Campillo B et al. Epidemiology of severe hospital-acquired infections in patients with liver cirrhosis: effect of long-term administration of norfloxacin. Clin Infect Dis. 1998;26(5):1066-70.

41. Runyon BA, AASLD. Introduction to the revised American Association for the Study of Liver Diseases Practice Guideline management of adult patients with ascites due to cirrhosis 2012. Hepatoloygy. 2013;57(4):1651-3.

42. Piano S et al.; International Club of Ascites Global Study Group. Epidemiology and effects of bacteria infections in patients with cirrhosis worldwide. Gastroenterology. 2019;156(5):1368-80.e10.

43. Elfert $A$ et al. Randomized-controlled trial of rifaximin versus norfloxacin for secondary prophylaxis of spontaneous bacterial peritonitis. Eur J Gastroenterol Hepatol. 2016:28(12):1450-4.

44. Shamseya MM, Madkour MA Rifaximin: a reasonable alternative for norfloxacin in the prevention of spontaneous bacterial peritonitis in patients with $\mathrm{HCV}$-related liver cirrhosis. Alexandria J Med. 2016;52(3):219-26.

45. Goel A et al. Systematic review with meta-analysis: rifaximin for the prophylaxis of spontaneous bacterial peritonitis. Aliment Pharmacol Ther. 2017:46(11-12):1029-36.

46. Lutz $P$ et al. Impact of rifaximin on the frequency and characteristics of spontaneous bacterial peritonitis in patients with liver cirrhosis and ascites. PloS One. 2014;9(4):e93909.

47. Kalambokis $\mathrm{GN}$ et al. Rifaximin improves systemic hemodynamics and renal function in patients with alcohol-related cirrhosis and ascites. Clin Gastroenterol Hepatol. 2012;10(7):815-8.

48. Vlachogiannakos $\mathrm{J}$ et al. Intestinal decontamination improves liver haemodynamics in patients with alcohol-related decompensated cirrhosis. Aliment Pharmacol Ther. 2009;29(9):992-9.

49. Vlachogiannakos J etla. Longterm administration of rifaximin improves the prognosis of patients with decompensated alcoholic cirrhosis. J Gastroenterol Hepatol. 2013;28(3):450-5.

50. Brown EL et al. Pretreatment of epithelial cells with rifaximin alters bacterial attachment and internalization profiles. Antimicrob Agents Chemother. 2010;54(1):38896.

51. Sartor RB. Review article: the potential mechanisms of action of rifaximin in the management of inflammatory bowel diseases. Aliment Pharmacol Ther. 2016:43(Suppl 1):2736.

52. Pimentel $M$ et al. ACG Clinical Guideline: small intestinal bacterial overgrowth. Am J Gastroenterol. 2020;115(2):165-78.

53. Gatta L, Scarpignato C. Systematic review with meta-analysis: rifaximin is effective and safe for the treatment of small intestine bacterial overgrowth. Aliment Pharmacol Ther. 2017;45(5):604-16.

54. Zhang $Y$ et al. Effects of SIBO and rifaximin therapy on MHE caused by hepatic cirrhosis. Int J Clin Exp Med.
2015;8(2):2954-7

55. Flamm SL et al. Rifaximin has the potential to prevent complications of cirrhosis. Ther Adv Gastroenterol. 2018;11:1756284818800307.

56. Salehi $S$ et al. Rifaximin reduces the incidence of spontaneous bacterial peritonitis, variceal bleeding and allcause admissions in patients on the liver transplant waiting list. Aliment Pharmacol Ther. 2019;50(4):435-41.

57. Bajaj JS. Review article: potential mechanisms of action of rifaximin in the management of hepatic encephalopathy and other complications of cirrhosis. Aliment Pharmacol Ther. 2016;43(Suppl 1):1126

58. Hudson $M$ et al. The impact of rifaximin-a on the hospital resource use associated with the management of patients with hepatic encephalopathy: a retrospective observational study (IMPRESS). Frontline Gastroenterol. 2017;8(4):243-51.

59. Orr JG et al. The impact on hospital resource utilisation of treatment of hepatic encephalopathy with rifaximin-a. Liver Int. 2016;36(9):1295303.

60. Patel VC, Williams R. Antimicrobia resistance in chronic liver disease. Hepatol Int. 2020;14(1):24-34

61. O'Connor JR et al. Rifampin and rifaximin resistance in clinical isolates of Clostridium difficile. Antimicrob Agents Chemother. 2008;52(8):2813-

62. Reigadas E et al. Breakthrough Clostridium difficile infection in cirrhotic patients receiving rifaximin Clin infect Dis. 2018;66(7):1086-91 